Chimia 46 (1992) 433-440

(C) Neue Schweizerische Chemische Gesellschaft ISSN $0009-4293$

\section{Das Berufsbild des Chemikers im Wandel der Zeit}

\author{
Tobias Studer*
}

(Überarbeitete Fassung eines Vortrags gehalten anlässlich der 73. Generalversammlung des Schweizerischen Chemiker-Verbandes am 21. April 1992 in Genf)

\section{Einleitung}

'Gentechnologie wird noch jahrzehntelang nicht viel bringen!' Dieser Satz wurde 1982, d.h. vor genau zehn Jahren, von einem erfolgreichen Chemiker ausgesprochen, der die Entwicklung der Chemie während eines halben Jahrhunderts aktiv miterlebt hatte. Dabei hatte er sich zwar an das rasante Entwicklungstempo in Wissenschaft und Industrie gewöhnt. Wenn er dieses jedoch in die Zukunft projizierte, wollte er erste wirtschaftliche Erfolge der Gentechnologie erst tief im 21. Jahrhundert ansetzen. In Wirklichkeit war und ist das Entwicklungstempo der Chemie eben nicht nur rasant, sondern auch progressiv. Dies bezieht sich nicht nur auf die Zahl der bekannten chemischen Verbindungen und Reaktionen sowie die Flut der Veröffentlichungen, sondern manifestiert sich insbesondere auch in der beängstigend sinkenden Halbwertszeit chemischen Wissens und der sinkenden Nutzungsdauer wissenschaftlicher Instrumente.

Dass ein Chemiestudent noch in den 50er Jahren einen grossen Teil seines Wissens aus dem damals mehr als ein halbes Jahrhundert alten und in 34. Auflage vorliegenden Gattermann schöpfte, ist heute kaum noch vorstellbar. Fast noch mehr Fantasie braucht es, um nachvollziehen zu können, dass noch in der Jahrhundertmitte in der chemischen Forschung gravimetrische Analysen mit Hilfe 40 Jahre alter Analysewaagen durchgeführt wur-

\footnotetext{
*Korrespondenz: Prof. Dr. T. Studer Wirtschaftswissenschaftliches Zentrum (WWZ) der Universitit Basel Institut für Betriebswirtschaft Petersgraben 51 CH-4051 Basel
}

den. Heute sieht sich ein potentieller Lehrbuchautor mit dem Risiko konfrontiert, dass sein Werk in der Zeit von der Manuskripterstellung bis zur Auslieferung veralten könnte, ein Forscher mit dem Risiko, dass ein wissenschaftliches Gerät zwischen Bestellzeitpunkt und Inbetriebnahme obsolet werden könnte. So munkelt man denn gelegentlich von Apparaturen, die zwar noch angeliefert, aber gar nicht mehr ausgepackt werden, sondern direkt im Keller verschwinden, bis die Anstandsfrist für eine ordentliche Entsorgung abgelaufen ist.

Genau so rasant wie die Chemie selbst hat sich auch das Berufsbild des Industriechemikers in diesem Jahrhundert gewandelt. In den letzten 10 Jahren hat sich der Autor mit einer grossen Zahl von Industriechemikern ausführlich über die Sonnen- und Schattenseiten des Chemikerberufes unterhalten. Über 100 dieser meist mehrstündigen Gespräche wurden detailliert aufgezeichnet, so dass eine faszinierende Dokumentation entstand, auf welcher die nachfolgenden Ausführungen im wesentlichen basieren. Der älteste Gesprächspartner wies Jahrgang 1900 auf, der jüngste 1962, so dass die berufliche Wirklichkeit des Industriechemikers ab ca. 1925 bis 1992 aufgrund von unbeschönigten Informationen aus erster Hand beschrieben werden können. Dabei werden etwas vereinfachend drei Chemikergenerationen unterschieden, nämlich eine ältere Generation, die noch vor dem 2. Weltkrieg in die industrielle Praxis einstieg, eine mittlere Generation, die in den Boomzeiten vom 2 . Weltkrieg bis zu den frühen 70er Jahren ihr Berufsleben begann, und eine jüngere Generation, die in der härter werdenden Zeit seit der Ölkrise zur chemischen Industrie stiess.

\section{Das Berufsbild der älteren Chemikergeneration}

\subsection{Herkunft und Berufswahl}

Die ältere Chemikergeneration stammte noch fast ausschliesslich aus gehobenen gesellschaftlichen Kreisen. War Studieren an und für sich schon teuer und für Angehörige aus wirtschaftlich schwächeren Kreisen kaum erschwinglich, so blieb ein Chemiestudium - wegen der teuren Chemikalien und Geräte mindestens doppelt so teuer wie ein sonstiges naturwissenschaftliches Studium - erst recht einer kleinen Schicht von Interessenten offen. Die Gesamtkosten eines Chemiestudiums entsprachen in etwa zwei bis drei Jahresgehältern eines Industriearbeiters. Für eine Chemikerlaufbahn kamen daher primär Söhne von Unternehmern, höheren Beamten, Ärzten und Juristen in Frage. Ihrer sozialen Herkunft entsprechend bildeten sie noch vor der Aufnahme des Studiums eine gesellschaftlich homogene privilegierte Gruppe.

Viele angehende Chemiker trafen und daran hat sich bis heute nichts geändert-erstens ihre Berufswahl relativ früh, z.B. mit 12-16 Jahren, und zweitens ohne klare Vorstellung hinsichtlich ihrer späteren Tätigkeit. Damals war es allerdings noch völlig unvorstellbar, dass ein Gymnasiast oder Student zum Zweck der Informationsbeschaffung einen Betrieb der chemischen Industrie besichtigen konnte. Für die jüngere Chemikergeneration war dies zwar bereits möglich, aber zwischen Berufswahl und Studienabschluss konnte sich so viel ändern, dass diese Informationen aus erster Hand trotzdem rasch obsolet werden konnten.

\subsection{Studium}

Das Chemiestudium war im ersten Drittel unseres Jahrhunderts das einzige im ganzen naturwissenschaftlichen Bereich, welches überhaupt Aussichten auf eine berufliche Tätigkeit ausserhalb der Lehre bot. Für Mathematiker, Physiker, Biologen, Mineralogen etc , hatte die Wirtschaft keinen Bedarf. Wer sich zu den Naturwissenschaften im weitesten Sinn hingezogen fühlte, landete nach rationaler Einschätzung späterer Arbeitsmöglichkeiten fast zwangsläufig beim Hauptfach Chemie. Demzufolge war das Chemiestudium auch das meistfrequentierte im naturwissenschaftlichen Bereich.

Das Studium selbst war meist kurz und bündig. Bis zur Promotion vergingen sieben bis zehn Semester. Vier Jahre galten als 'vernünftige' Studiendauer, und dies sowohl an Universitäten wie auch an der ETH. Vorlesungen im Hauptfach gab es relativ wenige. Um die Studienzeit zu füllen, verschrieb man den Studenten viele Vorlesungen aus benachbarten Gebieten, wobei Physik und Mathematik noch keinen direkten Bezug zur Chemie hatten und vielfach als unnötiger Ballast empfunden wurden. Während des Studiums blieb noch genug Raum für Vorlesungen aus anderen 
Fakultäten sowie ein bis zwei Auslandsemester, vorwiegend an deutschsprachigen Universitäten, da Chemie noch eine fast rein deutsche Wissenschaft war. Mit angelsächsischer Literatur kam der Student - wenn überhaupt - nur während der Dissertation in Kontakt. Englisch musste er daher nicht können. Die chemische Industrie nahm bereitwillig darauf Rücksicht, indem sie noch bis in die 50er Jahre hinein Übersetzer für englische Fachliteratur beschäftigte.

In den höheren Semestern bestand das Studium nur noch aus rein handwerklicher Labortätigkeit. 'Labor von 8-12 und 2-4 Uhr und hernach gesellige Freizeit' erinnert sich ein Zeitzeuge. Vor allem wurde viel gefeiert und gebechert. In der Rückschau war das Studium für die meisten 'froh und gemütlich'. Auch prüfungstechnisch war es nicht eigentlich schwierig, denn Prüfungen (ausschliesslich mündliche) gabs nur am Schluss und 'man fiel sowieso nicht durch'. Auf einen Professor entfielen höchstens wenige Dutzend Studierende, so dass sich in der Regel bis zur Prüfung auch persönliche Beziehungen ergaben, die ein Durchfallen eher unwahrscheinlich machten.

Für die Dissertation war bis zu den 20er Jahren mit einem zeitlichen Aufwand von Wochen oder Monaten zu rechnen. Im Vorwort einer solchen Arbeit aus jener Zeit findet sich denn auch der bemerkenswerte Hinweis, die vorliegende Dissertation sei in den Sommerferien anlässlich eines Aufenthaltes auf dem Landgut des verehrten Doktorvaters entstanden. Aus heutiger Sicht würde man denn auch viele damaligen Dissertationen höchstens als Seminararbeiten einstufen. Trotzdem machten sie Sinn, da sie in der Regel von klaren Aufgabenstellungen ausgingen und den Kandidaten zu selbständigem Arbeiten zwangen. Letzteres gewöhnte man ihm in den 30er Jahren eher wieder $a b$, indem sich zuerst an der ETH, dann auch an den Universitäten ein völlig neues, studienverlängerndes Dissertationskonzept entwickelte, bei welchem der Kandidat als rein handwerkliche Hilfskraft für umfangreiche Forschungsprojekte des Professors eingesetzt wurde, in die er selbst gelegentlich nur einen nebulösen Einblick hatte. Er arbeitete mehrere Jahre oft ohne jeden eigenen Spielraum nach detailliertesten Anleitungen des Doktorvaters, bis dieser das erlösende Wort sprach: 'Sie können jetzt zusammenschreiben'. Daraus entstanden nicht selten sehr heterogene Dissertationen ohne eigentliches Thema mit dem ominösen Titel 'Zur Kenntnis von ...'. Was allenfalls an wissenschaftlich brauchbarer Erkenntnis angefallen war, erschien in Publikationen des Doktorvaters.

\subsection{Eintritt in das Berufsleben}

In den 20er Jahren konnte der angehende Chemiker in der Regel bereits mit 24 Jahren seinen Doktorhut aufsetzen und sein Berufsleben in Angriff nehmen. Gegen Ende der 30er Jahre war er zu diesem Zeitpunkt jedoch oft schon 25-27 Jahre alt. Viel schneller schafften es die Absolventen eines Technikums. Sie gelangten im Extremfall bereits mit 18 Jahren auf den Markt, hatten aber bis gegen Ende der 30er Jahre in der Schweiz in der Regel noch keinen Zugang zur chemischen Grossindustrie. Ihr typisches Berufsfeld waren die Färbereibetriebe.

Wie kam nun ein frisch promovierter Hochschulchemiker zu seiner ersten Stelle? Der Arbeitsmarkt für Chemiker war in den 20er und 30er Jahren generell sehr zäh. Die Nachfrage war vor allem zahlenmässig bescheiden. Roche z.B. beschäftigte Mitte der 20er Jahre etwa zwei Dutzend Chemiker. Auf dem ganzen Platz Basel waren es knapp über 100 und der Einstellungsbedarf der ganzen Basler chemischen Industrie lag in einem typischen Jahr näher bei fünf als bei zehn. Das war sehr wenig im Vergleich zum jährlichen Output der schweizerischen Hochschulen. Daher brauchte ein Studienabgänger etwas Glück, um in der chemischen Industrie unterzukommen. Was mit den vielen geschah, die es nicht schafften, ist leider nie erforscht worden. Wer es jedoch schaffte, hatte entweder persönliche Beziehungen zu massgebenden Persönlichkeiten oder er kam durch Vermittlung seines Doktorvaters zu einer Stelle.

Zufälligkeiten bewirkten dabei bedeutsame berufliche Weichenstellungen: Katholiken und Bündner landeten in der Regel bei Ciba, Protestanten, Welsche und Karrer-Schüler bei Sandoz, RucizckaSchüler und Juden bei Roche. Professoren hatten oft ein Interesse daran, ihre Schüler in bestimmte Firmen zu lenken, da sie eine allzu grosse Streuung ihres Know-hows vermeiden wollten. So scheiterte ein Rucizcka-Schüler, der aus eigenem Antrieb zur Geigy wollte, am Veto seines Lehrers, der die Anstellung hintertrieb und den Kandidaten an Roche vermittelte.

Die Einstellung eines neuen Chemikers war für die damals nach heutigen Vorstellungen noch mittelgrossen Betriebe der Basler chemischen Industrie stets ein bedeutsames Ereignis. Der Chemiker wurde daher vom obersten Firmenchef persönlich empfangen und eingestellt. Bei Roche war allenfalls noch die graue Eminenz der Firma, Barells Sekretärin Dora Bider anwesend, die gelegentlich das letzte Wort betreffend Einstellung gesprochen haben soll. Es herrschten demnach Verhältnisse, wie wir sie heute noch bei einer mittelgrossen Bauunternehmung vorfinden könnten. $\mathrm{Zu}$ verhandeln gab es nichts. Die Anstellungsbedingungen wurden dem Kandidaten einfach mitgeteilt.

Das Anfangssaläreines Hochschulchemikers lag in den 20er und 30er Jahren im Bereich von $350-500$ Fr., meist jedoch nahe bei $450 \mathrm{Fr}$. Dies entsprach zwar dem Doppelten eines Chemiearbeiterlohnes, erlaubte jedoch noch keinen standesgemässen Lebensstil mit Familie. Salärabweichungen nach oben kamen nur bei Vorliegen besonderer Umstände vor, so etwa, wenn der Kandidat im Ausland bei einem besonders berühmten Professor gearbeitet hatte und Know-how mitbrachte, welcher in der Schweiz sonst nicht verfügbar war. Bewährte sich der junge Chemiker, stieg sein Einkommen rasch und nachhaltig an und konnte sich in wenigen Jahren real vervielfachen, insbesondere wegen der damals noch üblichen Erfolgsbeteiligung. DerChemiker stellte sich damit etwa von seinem 30. Lebensjahr an materiell gut bis sehr gut.

Am ersten Arbeitstag wurde der junge Chemiker sämtlichen Kollegen und damit automatisch auch allen Führungskräften vorgestellt, wozu nicht mehr als ein halber Morgen erforderlich war. Er gehörte damit vom ersten Tag an zum engen Kreis der Firmenelite, welche die Interessen ihres Berufsstandes wirksam zu wahren wusste. Und dann begann sein industrieller Alltag.

\subsection{Berufsalltag}

Von seiner Ausbildung her war der Chemiker damals ein handwerklich orientierter Generalist. Er war mit den gängigen präparativen Verfahren der organischen Chemie vertraut und konnte für sämtliche Tätigkeitsbereiche der chemischen Industrie eingesetzt werden: Farben, technische Chemikalien, Textilhilfsstoffe oder Pharmazeutika. Eine funktionelle Spezialisierung nach Forschung und Produktion war zunächst noch unuiblich. Es war damals noch selbstverständlich, dass jeder Chemiker selbständig nach neuen Verbindungen und Verfahren suchte, dabei Analysen selber durchführte, allfällige Patente selber anmeldete, Produktionsverfahren selber ausarbeitete und die Produktion 'seiner' Produkte selber betreute. Letzteres war ja das eigentliche Berufsziel. Nicht die Produktion von Wissen, sondern von Handelsprodukten war seine Aufgabe.

Der Begriff 'Forschung' kam in der chemischen Industrie erst nach dem 2. Weltkrieg auf. Vorher machte man eben schlicht 'Chemie', ohne sich als Forscher zu empfinden. Die primär handwerkliche Tätigkeit wie Glasblasen, Holz und Metall bearbeiten, Apparaturen basteln, Kork bohren, Schmelzpunkte bestimmen etc. 
hatte auch nichts mit dem jeweils aktuellen Geschehen an der wissenschaftlichen Front zu tun. Von dieser war der praktisch tätige Industriechemiker weit entfernt. Die Bücher, die ihn durch das Studium begleitet hatten, dienten ihm auch in der Praxis noch jahrzehntelang. Originalarbeiten las er kaum. Zur Kenntnis nahm er allenfalls die neuesten Patente in seinem Arbeitsgebiet. An den Besuch von Weiterbildungsveranstaltungen oder Kongresssen dachte er nicht im Traum.

Eine innerbetriebliche Information war vollkommen inexistent. Aus Gründen der Diskretion arbeitete jeder Chemiker völlig einsam und ohne jeden fachlichen Kontakt zu Kollegen, wobei Doppelspurigkeiten in Kauf zu nehmen waren. Sie waren anderseits auch ein wirksamer Motivator: Wer zuerst kam, erntete zuerst und kassierte die Erfolgsbeteiligung. Der potentielle Erfinder musste stets befürchten, von einem Kollegen in einem benachbarten Labor überrundet zu werden, was zu maximalem Arbeitstempo unter $\mathrm{Ge}$ heimniskrämerei zwang. Gelegentlich wusste nicht einmal der Vorgesetzte, was im Labor eines Chemikers lief. Der Chemiker war im übrigen der einzige Geheimnisträger, da Arbeiter und Laboranten nicht die geringste Ahnung von Chemie hatten. Man liess sie absichtlich und zum Teil mit abstrusen Massnahmen, z.B. Geheimcodes anstelle von Stoffbezeichnungen, falsch kalibrierten Thermometern etc. im Ungewissen über das, was sie zu tun hatten. Mit chemischen Formeln kamen sie prinzipiell nicht in Berührung.

Neuentwicklungen entsprachen damals nicht Zielvorgaben des Marketings oder einer koordinierenden Forschungsplanung, sondern gingen von punktuellen Überlegungen des individuellen Chemikers aus. Erst wenn er ein neues Produkt zur Handelsreife gebracht hatte, wurde die kaufmännische Abteilung orientiert. Dazu verriet ein Zeitzeuge: 'Das war gar nicht schlecht, man vermied damit jedes unnütze Palaver.'

Dem handwerklichen Charakter der Tätigkeit des Chemikers entsprechend hatten die Labors eher den Charakter einer Werkstatt als eines wissenschaftlichen Arbeitsraumes. Ältere Photographien solcher Labors haben denn auch eine gewisse Ähnlichkeit mit der Werkstatt eines heutigen Schreiners, Glasers oder Schuhmachers. Sie waren nach heutigen Massstäben unglaublich primitiv. Von Zeitzeugen werden sie als 'schauderhafte Höhlen oder Verschläge' beschrieben. Einem neu eintretenden jungen Chemiker wurde dabei selbstverständlich das schlechteste Labor auf dem ganzen Werkgelände zugeteilt. Einziger Komfort war allenfalls eine lärmige Riementransmission. Im Winter musste er am Morgen zuerst die Wasserleitungen auftauen. Gewogen wurde mit ungedämpften Waagen, die notdürftig gegen Durchzug geschuitzt wurden. Kapellen waren noch Luxus. Es wurde als durchaus normal empfunden, wenn ein Chemiker beim offenen Arbeiten mit Äthylenbromid betäubt wurde und verreiste, wenn er bei einer Wasserdampfdestillation von Allylbromid für ein paar Stunden erblindete oder sich beim Arbeiten mit $a$ Nitrophenol vorübergehend vergiftete. 100-Kiloansätze zur Herstellung von Benzidin erfolgten offen und ohne jede Vorsichtsmassnahme, wobei hernach die Nutschen mit blossem Finger ausgeputzt wurden.

Primitive Labors der geschilderten Art reichten dennoch für eine erfolgreiche Tätigkeit des Chemikers aus, da sie den bescheidenen Anforderungen durchaus genügten. Die Welt des Chemikers beschränkte sich noch auf die drei Dimensionen Temperatur, $\mathrm{pH}$ und Agens. Thermometer und Lackmuspapier waren neben der Nase und dem Auge des Chemikers, gelegentlich auch seiner Zunge und seines Magens, die einzigen verfügbaren Sensoren. Druckanwendung und Druckmessung spielten noch kaum eine Rolle. Im Vordergrund stand die Chemie wässriger Lösungen in offenen Gefässen, d.h. bei Atmosphärendruck. So wurde insbesondere auch produziert, und zwar z.T, noch im Freien.

Erst ab der zweiten Hälfte der 30er Jahre und in den 40er Jahren wurden sukzessive zeitgemässe und komfortable Labors eingerichtet. Unmittelbarer Anlass war aber nicht die Rücksicht auf die Mitarbeiter, sondern die Entwicklung der pharmazeutischen Chemie in Richtung Naturstoffe und damit zwangsläufig in Richtung Mikrochemie. Das Arbeiten im Milligrammbereich war in den alten, primitiv eingerichteten und staubigen Labors gar nicht möglich, sondern erforderte eine völlig neue technische Umgebung. Erst jetzt kamen auch zaghaft zeitgemässe, an Hochschulen und sogar Techniken bereits eingeführte Errungenschaften wie Schliffapparaturen, pH-Meter, Infrarotspektroskopie oder gar Hochvakuumpumpen auf. Für normale Anwendungen hielt man solche Errungenschaften zwar noch lange als Luxus. Noch Ende der 50er Jahre erforderte die Beschaffung einer Schliffapparatur bei $S a n d o z$ das spezielle Visum eines Vizedirektors.

Der Einzug zeitgemässer Technik in das Labor des Chemikers brachte auch das Ende des Universalchemikers. Das Aufkommen der pharmazeutischen Sparte hatte schon zuvor zum Einsatz von Chemikern in einem weit entfernten neuen Berufsfeld geführt: der Ärztepropaganda.
Jetzt wurden erstmals bereits spezialisierte Chemiker wie z.B. Mikroanalytiker eingestellt. Gleichzeitig begann sich die Differenzierung des Chemikerberufs in die beiden Richtungen Labor und Betrieb zu institutionalisieren.

Chemiker der älteren Generation hatten noch gute Chancen auf rasche Erfolge. Neue Farbstoffe, neue technische Chemikalien, ja selbst neue pharmazeutische Präparate liessen sich von einem Einzelforscher in vernünftiger Zeit entwickeln und einführen. Die Qualitätsanforderungen waren gering. Ein 10\%iger oder gar unbekannter Reinheitsgrad war kein Hindernis für die Markteinführung eines pharmazeutischen Präparates. An Nebenwirkungen nahm man praktisch alles in Kauf. Zwischen der Erfindung eines pharmazeutischen Wirkstoffes und der Markteinführung lagen im günstigsten Fall nur etwa drei Jahre. Im Farben- und Chemikalienbereich konnte es ein Chemiker im Verlauf seines Arbeitslebens durchaus auf einige Dutzend Handelspräparate bringen, im pharmazeutischen Bereich auf bis zu zehn. In der Regel hatte daher der Chemiker immer wieder persönliche Erfolgserlebnisse, die sich auch auf Karriere und Gehalt positiv auswirkten.

\subsection{Karriere und gesellschaftliche Stellung}

Im Normalfall brachte es der Chemiker der älteren Generation bis zum Vizedirektor, wobei dieser Titel noch nicht inflationär entwertet war, sondern auf eine absolute Respektsperson hindeutete. Kaum einer blieb auf der Stufe des Prokuristen stehen. Etliche machten allerdings noch wesentlich steilere Karrieren, da sich die Direktionen von Chemieunternehmungen bis zur Mitte des Jahrhunderts ausschliesslich aus Chemikern rekrutierten. Es war selbstverständlich, dass ein chemisches Unternehmen nur von Chemikern geleitet werden konnte. Bei BASF war dies sogar in den Statuten explizit verankert. Ein geflügeltes Wort jener Zeit lautete: 'Wenn man den Chemiker nicht totschlägt, wird er Direktor.'

Die Tatsache, dass damals praktisch jeder Chemiker automatisch in der Hierarchie aufstieg, war insbesondere Folge des gewaltigen Wachstumsschubs nach dem 2. Weltkrieg. Aus mittelgrossen Unternehmen wurden weltumspannende Konzerne. Wo vorher ein paar Dutzend Chemiker wirkten, waren es nun plötzlich Hunderte. Die Organisationen wucherten und es entstand ein gewaltiger Bedarf nach chargierten Kaderkräften. Die ihre Standesinteressen wirksam wahrnehmende kleine Chemikerelite der Vorkriegszeit, jetzt im richtigen Alter für Führungspositionen, stiess ohne nennenswerte Konkur- 
renz in das Vakuum vor und besetzte in gegenseitiger Abdeckung fast sämtliche Management-Positionen. Ob sie in jedem Fall für ihre neuen Aufgaben gerüstet war, blieb eine Frage von untergeordneter $\mathrm{Be}$ deutung. Die Ertragslage erlaubte jedes Mass an Ineffizienz.

Der Chemiker der älteren Generation war Zeit seines Arbeitslebens vom Nimbus des Übermenschen geprägt. In der Vorkriegszeit gab es in einem Unternehmen der chemischen Industrie eigentlich zwei völlig verschiedene Arbeitswelten. Auf der einen Seite war es die Masse der Arbeiter und Angestellten ohne eigentliche Berufsausbildung, die nahe beim Existenzminimum vegetierte, auf der anderen Seite die kleine, gut bezahlte Elite der Chemiker. Zwischen diesen beiden Welten bestand eine unendlich weite soziale Distanz. Insbesondere Betriebschemiker wurden bereits als Halbgötter empfunden. Sie hatten ein höheres Prestige und verdienten deutlich mehr als Laborchemiker, hatten unbeschränkte Macht über ihre Mitarbeiter und konnten sich jede Marotte leisten. Bei einem Direktor stellte sich höchstens die Frage, ob er auf der gesellschaftlichen Stufenleiter vor oder hinter dem Herrgott einzureihen war.

Die soziale Distanz zwischen Belegschaft und Chemikerkaste wurde auf vielfältige Art künstlich gefördert. Chemiker verfügten über eine Vielzahl von Standesprivilegien. Sie trugen als erste und einzige weisse Mäntel. Sie verfügten zunächst als einzige über eine Kantine, in welcher weiss gedeckt war und mit massivem Silberbesteck gegessen wurde. Sie verfügten über eigene Toiletten und Bäder. Sie erschienen grundsätzlich eine Stunde später zur Arbeit als die sonstige Belegschaft. Sie hatten als einzige vier Wochen Ferien. Und selbst in den Ferien musste die soziale Distanz gewahrt werden. Noch lange nach dem 2. Weltkrieg stellte z.B. Sandoz den Chemikern komfortable Ferienhäuser in La Punt zur Verfügung, während für Laboranten etwas schlichtere Wohnungen auf der Lenzerheide bereitstanden. Dass ein Chemiker und ein Laborant am selben Ort oder sogar unter dem selben Dach Ferien machen sollten, war nicht vorstellbar.

Soziale Distanz wurde nicht nur zwischen Belegschaft und Chemikerkaste künstl ich geschafffen, sondern mit der Zeit auch innerhalb der Chemikerkaste selbst, indem die Spitzenkräfte mit Hilfe spezieller Privilegien, mit aus heutiger Sicht teilweise peinlichem Personenkult und entsprechender Salarierung in weit entfernte Sphären entrückt wurden. Verdiente ein Hochschulchemiker vor dem 2. Weltkrieg zu Beginn seiner Laufbahn noch um die 5000 Fr. pro Jahr, soll ein Betriebsleiter beispielsweise mit bis zu 90000 und ein Direktor mit bis zu $300000 \mathrm{Fr}$. pro Jahr honoriert worden sein. Das Monatssalär entsprach damit dem Preis eines mittleren Einfamilienhauses. Spitzenerfinder brachten es wegen der Erfolgsbeteiligung zeitweise noch auf wesentlich höhere Einkommen. Für damalige Verhältnisse waren das unvorstellbare Summen, die allein schon wegen der Gefahr sozialer Spannungen um jeden Preis geheimgehalten werden mussten. So war es etwa Chemikern noch bis in die 30er Jahre hinein untersagt, Wohnsitz in einer Landgemeinde zu nehmen, weil zu befürchten war, dass die für die Steuern verantwortlichen nebenamtlichen Gemeinderäte nicht dichthalten würden.

Wollte man die damaligen Salär- und Kaufkraftrelationen auf die heutige Zeit übertragen und hätten sie nach wie vor Gültigkeit, so könnte ein junger Hochschulchemiker, der im Jahr 1992 mit einem Salär von vielleicht $85000 \mathrm{Fr}$. in die Praxis einsteigt, davon ausgehen, dass er mit etwas Glück auf dem Höhepunkt seiner Karriere durchaus 5 Millionen Fr. pro Jahr verdienen könnte.

Trotz Karriere waren fast alle Chemiker der älteren Generation bis zum letzten Tag ihres beruflichen Wirkens im Labor tätig. Wer mit 24 Jahren eintrat, konnte so 41 Jahre lang unablässig forschen. Auch nach der Pensionierung arbeitete der Chemiker gelegentlich noch halbtags oder als Consultant bis weit über den 70 . Geburtstag hinaus weiter. Einer im Minimum dreieinhalbjährigen Hochschulausbildung konnte demnach im Maximum annähernd ein halbes Jahrhundert praktische und unmittelbare Labortätigkeit folgen, so dass sich die Investition in ein Chemiestudium in jeder Hinsicht als ausserordentlich fruchtbar erwies. Bis zu 300 Patente, einige Dutzend Publikationen und einige Dutzend Handelspräparate, Aufstieg zum Vizedirektor, materieller Wohlstand und nach eigenen Plänen gebaute Villa mit $2000 \mathrm{~m}^{2}$ Umschwung an bevorzugter Lage waren eine nicht unübliche Ausbeute eines langen Chemikerlebens. Am zutreffendsten charakterisierte die berufliche Situation der älteren Chemikergeneration ein Betroffener selbst: 'Wir profitierten davon, dass es eigentlich 40 Jahre lang immer nur aufwärts ging.'

\section{Das Berufsbild der mittleren Chemikergeneration}

\subsection{Herkunft und Berufswahl}

Die mittlere Generation von Chemikern, die in der Zeit vom 2. Weltkrieg bis zum Ausbruch der Ölkrise in das Berufs- leben trat, stand zunächst unter dem beherrschenden Eindruck der Erfahrungen der älteren Generation, traf dann aber in ihrer Berufswirklichkeit auf völlig veränderte Rahmenbedingungen. Zum Teil hatte sie es leichter als die ältere Generation, z.T. aber auch erheblich schwerer.

Der Zugang zum Chemikerberuf war nach dem 2. Weltkrieg generell erleichtert. Dazu trugen eine Reihe von Faktoren bei: die deutlich gestiegene Maturandenquote, die verbesserte Einkommenssituation weiter Kreise der Bevölkerung, die relative Verbilligung des Chemiestudiums infolge industrieller 'Economy of scale'Effekte, welche die Preise für die meisten Chemikalien im Vergleich zur Vorkriegszeit auf einen Bruchteil senkten, ein deutlich verbessertes Stipendienwesen sowie nicht zuletzt der Übergang zum einheitlichen und existenzsichernden Doktorandensalär. Auch Frauen drängten nun in den Beruf. Vereinzelt gab es die Chemiestudentin zwar schon vor dem 2. Weltkrieg; sie kam aber damals für einen Einsatz in der chemischen Industrie grundsätzlich nicht in Frage. Die Zahl der Chemiestudierenden entwickelte sich zwar positiv, jedoch unterdurchschnittlich, was nicht zuletzt auch auf verbesserte Berufsaussichten in anderen naturwissenschaftlichen Disziplinen zurückzuführen war. Man denke dabei etwa an den Physikerboom im Zusammenhang mit dem Aufkommen der Atomenergie.

An der sozialen Herkunft der Chemiker änderte sich zunächst wenig. Nach wie vor stammte der Chemiestudent praktisch ausschliesslich aus Unternehmer- oder Akademikerkreisen. Vereinzelt fanden auch Angehörige des unteren Mittelstandes, z.B. Söhne von Handwerkern, Landwirten und kaufmännischen Angestellten Zugang zum Chemikerberuf. Dass der Sohn eines Chemiearbeiters Chemiker wurde, war die absolute Ausnahme. Es ist dem Autor denn auch aus jener Zeit nur ein einziger derartiger Fall bekannt.

Den positiven Erfahrungen der älteren Chemikergeneration zum Trotz studierten Söhne von Chemikern kaum je Chemie. Eine der seltenen Ausnahmen hatte dafür eine uiberraschend einfache Erklärung zur Hand: 'Es ist eben abschreckend, wenn der Vater immer stinkend nach Hause kommt'. Bei den primitiven Arbeitsbedingungen der älteren Chemikergeneration galt ausnahmsweise das Motto: pecunia olet.

Die Berufswahl erfolgte beim Chemiker der mittleren Generation im wesentlichen nach denselben Mechanismen wie bei der älteren. Es gab viele Frühentschlossene, die schon in jungen Jahren mit damals noch frei zugänglichen chemischen Substanzen gebastelt hatten, wobei Rake- 
ten, Knallgas und Schwarzpulver immer noch die selbe Faszination ausübten. Neu war allenfalls der Zugang zur Chemie via Photographie und Dunkelkammer oder via Flüssigkeitswiderstände zum Steuern einer elektrischen Eisenbahn. Die Informationsbasis über die industrielle Wirklichkeit des Chemikerberufs war nach wie vor extrem dürftig. Zwar führte bereits Ende der 50er Jahre die Firma Plüss Stauffer Schnupperlehren für Gymnasiasten durch, eine Idee, die in der Branche jedoch nicht Schule machte.

Die günstigen wirtschaftlichen Aussichten spielten damals bei der Wahl eines Chemiestudiums eine wichtige Rolle. 'Die meisten studierten im Glanz der Sonne der Chemiebarone' gibt ein Vertreter der mittleren Chemikergeneration in der Rückschau unumwunden zu. Das Resultat waren oft mittelmässige Chemiker ohne eigentliche Berufung zur Chemie. Nicht zuletzt war auch das Prestige des Chemikers noch unangeschlagen. Auf der Beliebtheitsskala figurierte er ganz oben, noch deutlich vor dem Mediziner, was nur teilweise auf die damalige wirtschaftliche Situation des Chemikers zurückzuführen war. Zum Teil basierte das Prestige auf der Tatsache, dass der Chemiker in den Augen weiter Bevölkerungsschichten jener Mann war, der aufgrund rätselhafter, nicht nachvollziehbarer, aber jedenfalls genialer Überlegungen neue Heilmittel gegen gefährliche Krankheiten 'erfand' und damit als 'Helfer der Menschheit' empfunden wurde.

\subsection{Studium}

Die gewaltigen Schübe, welche die Entwicklung der Chemie zwischen den $30 \mathrm{er}$ und den 50er Jahren kennzeichneten, blieben zunächst ohne angemessene Auswirkung auf die Ausbildung. Die Professoren selbst mussten nach dem Krieg den Rückstand zur angelsächsischen Forschung zuerst aufarbeiten. Dabei war es verständlich, dass sie ihn zuallererst im Bereich der Forschung zu überwinden trachteten, was zu einer teilweisen Vernachlässigung der Lehre führte. Die Ausbildung der jungen Chemiker unterschied sich daher inhaltlich und methodisch nach dem Krieg noch längere Zeit nicht wesentlich von der Vorkriegsausbildung. Die jahrzehntealten Riten der analytischen Ionentrenngänge, schon längst ohne Bezug zur industriellen Wirklichkeit und weitgehend zum Selbstzweck resp. zur billigen Beschäftigungstherapie verkommen, sowie die Darstellung von Präparaten nach zum Teil ebenfalls jahrzehntealten Rezepten füllten einen grossen Teil des Laborpensums aus. Mit der modernen Chemie kam der Student eigentlich erst während seiner Dissertationszeit in direkte Berührung.
Als Konsequenz entwickelte sich die Promotionszeit zum Rückgrat einer Chemikerausbildung. War eine Dissertation für den Chemiker der älteren Generation oft noch so etwas wie eine praktische Prüfung als krönender Abschluss eines Studiums gewesen, wie heute etwa noch bei den Medizinern, stellte nun die Nachdiplomausbildung eine hochspezialisierte und gelegentlich etwas zufällige Hauptphase des Studiums dar, welche die eigentliche Ausbildungszeit bis zum Diplom faktisch zum Propädeutikum degradierte. Demzufolge hatte ein lediglich diplomierter Chemiker auf dem Arbeitsmarkt kaum Chancen. Als Nebeneffekt ergab sich das Risiko, dass ein Chemiestudent jahrelang in einem wenig relevanten Hobbygebiet eines Professors 'verplemperte'. Die Ausbildungszeit konnte sich bis zu fast abschreckenden Horizonten verlängern. Es gab Professoren, bei denen Promotionszeiten von fünf Jahren normal, solche von sechs Jahren nicht unüblich waren. Das Chemiestudium kam in den Ruf, hart und lang zu sein, was sich auf die nachfolgende Generation alles andere als stimulierend auswirkte.

Eine zusätzliche Verlängerung der Chemikerausbildung brachte auch das gegen Ende der 50er Jahre faktisch zum Obligatorium gewordene Postdoktorat in den USA. Dieses wurde von der Industrie zunächst nur gewünscht, dann als Voraussetzung zumindest für einen Einsatz in der Forschung betrachtet und gelegentlich auch vom späteren Arbeitgeber finanziert. Aus Sicht des jungen Chemikers ersetzte das Postdoktorat die früher obligaten Auslandsemester während des Studiums und stellte oft die einzige Möglichkeit dar, zwischen dem Studium und der 'Endstation Industrie' ein oder zwei Wanderjahre einzuschalten.

\subsection{Eintritt in das \\ Berufsleben}

Die mittlere Generation von Chemikern gelangte im Durchschnitt wesentlich später auf den Arbeitsmarkt, typischerweise mit etwa 28 bis 31 Jahren, einem Alter, in welchem die ältere Generation in der Regel bereits die Grundlagen für ihre Karriere gelegt hatte.

Die Situation am Stellenmarkt war für die mittlere Chemikergeneration die beste unseres Jahrhunderts. Nach dem 2. Weltkrieg entwickelte sich die schweizerische chemische Industrie in nie erwartetem Tempo. Der Bedarf nach Chemikern war erheblich und überstieg gelegentlich die inländische Produktion. Stockungen gab es nur kurzfristig, so etwa Ende der 40er und Anfang der 50er Jahre. Ab Mitte der 50er Jahre stieg die Nachfrage jedoch unaufhaltsam an und in den 60er Jahren wurden die Studienabgänger förmlich umworben. Das offene Stelleninserat löste das zuvor übliche chiffrierte ab. 'Ich schrieb zehn Briefe und erhielt acht konkrete Stellenangebote' vermeldet ein Vertreter dieser mittleren Chemikergeneration.

Der junge Chemiker war jetzt in der komfortablen Lage, seine erste Stelle unter mehreren echten Alternativen auswählen und damit, im Gegensatz zum Chemiker der älteren Generation, erheblichen Einfluss auf die Richtung seines beruflichen Werdegangs nehmen zu können. Er konnte jetzt sogar über das Anfangssalär verhandeln. Professoren kassierten fünfstellige Beträge für die Vermittlung eines Chemikers. Selbst schlechte Chemiker oder Ausländer im Alter von 50 oder mehr Jahren wurden eingestellt. Es war ein wahrer Wettlauf unter den Chemiefirmen. Trotzdem blieben die Anfangssaläre kaufkraftmässig eher bescheiden. Mit rund 750 Fr. im Jahr 1950 und 3000 Fr. im Jahr 1970 entsprachen sie, wie schon vor dem zweiten Weltkrieg, in etwa dem Doppelten eines Chemiearbeiterlohnes.

Der Hunger der Industrie nach Chemikern war in jener Zeit so gross, dass jetzt auch selbstauferlegte Schranken fielen. Zwar suchte man nach wie vor in erster Linie Hochschulchemiker. Mangels Masse stellte man jetzt aber auch HTL-Chemiker in grösserer Zahl ein. Diese waren nach dem Krieg oft besser ausgebildet als Hochschulchemiker aus der Vorkriegszeit, was zu Spannungen führen konnte. Man 'schnitt' die Techniker und liess sie mit künstlich geschaffener Distanz spüren, dass sie nicht zur Elite gehörten. So gewährte man ihnen beispielsweise keinen Zutritt zur Bibliothek oder verlangte von ihnen, Anschaffungen im Wert von 2 Fr. durch einen promovierten Chemiker visieren zu lassen. Indessen: Qualität setzt sich durch. Nach einigen Jahren Berufspraxis stellte sich vielfach heraus, dass HTL-Chemiker auf bestimmten Gebieten Gleiches oder gar Besseres leisteten als Kollegen von der Hochschule. Sie machten, wenn auch zögerlich, Karriere und erkämpften sich Schritt für Schritt in einem etwa 20-jährigen Prozess eine faktische Gleichstellung.

Noch widerwilliger als HTL-Chemiker wurden ab ca. 1960 auch vereinzelte Hochschulchemikerinnen eingestellt. Sie kamen jedoch nur für spezielle Aufgaben, meist dienender Art, in Frage, so etwa als Literaturchemikerin, die ihren männlichen Kollegen das lästige Recherchieren abnehmen durfte, als Analytikerin für Routineaufgaben oder als Patentsachbearbeiterin. Ihre Aufstiegschancen waren damals exakt Null. Sie waren im wahrsten Sinn des Wortes 'Lückenbüsserinnen'. 


\subsection{Berufsalltag}

Chemiker der mittleren Generation wurden nicht mehr als Universalchemiker eingestellt mit dem schlichten Auftrag, 'Handelsprodukte zu erfinden'. Die inzwischen gross gewordenen Unternehmen der Basler chemischen Industrie wiesen jetzt einen hohen Grad an Arbeitsteilung auf. Der junge Chemiker wurde für eine spezielle, eng umschriebene Aufgabe im Rahmen eines unkontrolliert wuchernden Forschungs- und Produktionsapparates angeworben. Seine Einstellung erfolgte mit dem Ziel, ein ganz bestimmtes Kästchen in einem Organigramm zu füllen. Viele der angebotenen Aufgaben hatten nur noch am Rande mit Chemie zu tun, so dass Chemiker der mittleren Generation gelegentlich schon vom ersten Tag an ausserhalb eines Labors arbeiteten.

Es eröffneten sich jetzt jedoch auch völlig neue und faszinierende Einsatzmöglichkeiten, so etwa beim Aufbau neuer Tochtergesellschaften im fernen Ausland, wo junge Chemiker mit Generalvollmachten ausgestattet pionierhaft neue Fabriken aus dem Boden stampften und lokale Produktionen aufzogen. Wer zu Hause blieb, konnte allerdings bald an Grenzen seiner kompetenzmässig definierten Funktion stossen. 'Schlimm war's, wenn einer in der Gruppe Antirheumatika ein Analgetikum fand. Er graste damit im Gärtlein des Anderen' erinnert sich ein Betroffener. Ein zweiter fand zufällig eine Reaktion, die ein völlig neues Gebiet erschlossen hätte. Da dies nicht zu seinem Stellenbeschrieb gehörte, wurde er von seinem Chef zurückgebunden: 'Seien Sie zufrieden, Sie haben ja bereits ein Patent.'

Überhaupt scheint die Arbeitsweise jener Zeit nicht sonderlich innovationsfördernd gewesen zu sein, was sich im Nachhinein auch zahlenmässig belegen lässt. Zum Teil waren die allzu vielen Hierarchiestufen daran schuld, dass neue Ideen nicht bis zu den Entscheidungszentren vordrangen. Zum Teil war es aber auch die zurückhaltende Skepsis der an den Schalthebeln der Macht sitzenden älteren Generation gegenüber Neuentwicklungen. Für die ältere Generation waren die Naturstoffe das edelste und letzte Tummelfeld chemischen Strebens. Gegen die Beschäftigung mit Eiweissen hatte man Vorbehalte, denn das waren 'verachtenswerte undefinierbare Schleime' und damitein völlig minderwertiges Betätigungsfeld. 'Diese Substanzen haben nicht zu wenig, sondern zu viel Wirkung' beschied ein älterer Chef seinen jüngeren Mitarbeiter. Es ist daher kaum verwunderlich, dass unter jüngeren Chemikern gelegentlich eher passive Einstellungen um sich griffen. 'Wenn man in einer Grossfirma eine Entdeckung macht, bringt das niemandem etwas ausser Mehrarbeit' charakterisierte ein Betroffener resignierend die Situation.

Die Funktionalisierung und Spezialisierung betraf nicht nur das Wirkungsfeld des Chemikers, sondern jegliche Tätigkeit in der chemischen Grossindustrie. Waren die Chemiker als fast einzige Akademiker in der Vorkriegszeit noch weitgehend unter sich gewesen, wurden jetzt z.B. auch Juristen und Ökonomen, Mathematiker und Informatiker, Physiker und Biologen, Pharmakologen und Mediziner eingestellt. Der Chemiker verlor damit fast automatisch seine alte Sonderstellung. Er war nun einer von vielen und - schlimmer noch wurde von der Konkurrenz langsam aber sicher überholt. Es tat weh, realisieren zu müssen, dass jetzt ein Programmierer einen deutlich höheren Anfangslohn beziehen konnte als ein Chemiker mit zehnjähriger Ausbildung.

Da es jetzt in einer einzigen Unternehmung Hunderte von Chemikern gab, war jede Exklusivität dahin. Man kannte sich auch nicht mehr persönlich und bildete keine geschlossene Kaste mehr. Die mittlere Generation von Chemikern erlebte damit hautnah den Abstieg vom einsamen Podest und die Verdrängung des Chemikers aus wichtigen Führungspositionen durch Ökonomen und Mediziner. Die vielen alten Statussymbole und Vorrechte des Chemikers zerbröckelten im Verlauf der Zeit unaufhaltsam, und zwar in den seltensten Fällen durch gezielte Abschaffung, sondern passiv, indem die selben Vorrechte auch auf weitere Mitarbeiterkategorien ausgedehnt wurden, so etwa das Recht auf den weissen Mantel, das Recht, Bibliothek und Kantine betreten zu dürfen, die flexible Arbeitszeit, das Privileg des Monatslohns oder das Privileg der Versorgung mit Firmeninformation.

Technisch waren die Arbeitsbedingungen der mittleren Generation weit besser als diejenigen der älteren Generation. Dem Chemiker standen nun helle, modern eingerichtete Labors zur Verfügung. Servicestellen übernahmen zeitraubende Analysen oder Recherchen. Gut ausgebildete Laboranten erhöhten seine Arbeitsproduktivität erheblich. War es früher insofern zweckdienlich gewesen, einem Chemiker einen einzigen Laboranten zuzuordnen, als der Chemiker nur in bescheidenstem Umfang Botengänge und einfache Verrichtungen delegieren konnte, übernahmen nun Laboranten fast die gesamte eigentliche Labortätigkeit und verbannten den Chemiker als reinen Vordenker in seine kleine Zelle, die ursprünglich nicht für Daueraufenthalt konzipiert worden war. Ein Chemiker konnte nun soviel Arbeit delegieren, wie sein Gehirn an Ideen produzierte, so dass er auch mehrere La- boranten sinnvoll auslasten konnte. Anderseits wurde der Chemiker nun abhängig von der Qualität seiner Laboranten, indem diese oft über seinen Erfolg oder Misserfolg entschieden. Dies trug zur teilweisen Nivellierung des Gefälles zwischen Chemiker und Laborant bei, und zwar nicht nur salär-, sondern auch führungsmässig. Chemiker sprachen jetzt ihre Laboranten mit 'Sie' an und hatten Hemmungen, diese für einfache Botengänge einzuspannen.

Die stark angestiegene technische Arbeitsproduktivität brachte dem Chemiker selbst keine Vorteile, da gleichzeitig die Trefferwahrscheinlichkeit derchemischen Forschung stark rückläufig wurde. Zudem verlagerte sich der Engpass vom Labor zum Marketing oder zum Verkehr mit Behörden. Wo man früher für die Registrierung eines Heilmittels einfach 'einem berühmten Professor eine Tranche Bares im Gegenzug für eine Unterschrift zugesteckt' hatte, lieferte man jetzt Registrierungsunterlagen per Lastwagen an eine anonyme Behörde ab. Bis zu $90 \%$ seiner Arbeitszeit soll ein im übrigen erfolgreicher Chemiker der mittleren Generation für den Verkehr mit Gesundheitsbehörden aufgewendet haben. Der Qualität der Produkte war dies allerdings nur förderlich, da aus ökonomischen Gründen schon im Vorfeld einer aufwendigen Registrierung ausgesiebt wurde, was nicht wirklich erfolgversprechend war.

Das bestimmende Ereignis im Berufsleben des Chemikers der mittleren Generation war der unerwartet tiefe Einbruch im Gefolge der Ölkrise. Stagnierende oder gar rückläufige Umsätze, drastischer Margenzerfall und Gewinneinbruch, andauernde Reorganisationen, Plafonierungen, Zurücknahmen von Zielsetzungen und Budgets sowie teilweise völlig veränderte Prioritäten übten einen nachhaltigen Einfluss auf manche Chemikerlaufbahn aus. Viele von Chemikern wahrgenommene Funktionen wurden plötzlich überflüssig. Es wurde Speck abgebaut, der sich allerdings zuvor in reichlichem Mass angesetzt hatte. 'In meiner Abteilung arbeiten 60 Chemiker, 15 würden es auch tun' schätzte ein Betroffener seine Lage realistisch ein. Karrieren wurden plötzlich geknickt oder beendet, Saläre für viele Jahre eingefroren, Unglückliche mit 56 Jahren und halbem Salär in Zwangspension geschickt. Mancher fand sich plötzlich in einer Funktion, für die er eigentlich überqualifiziert war. 'Ich war der teuerste kaufmännische Angestellte' erinnert sich ein Vizedirektor, dessen Betrieb geschlossen wurde und der in einer Personalabteilung landete. Frustration, Rückzug ins Privatleben nach Büroschluss und Sehnsucht nach der Pension kamen auf. 


\subsection{Karriere und gesellschaftliche Stellung}

Das typische Curriculum des Chemikers der mittleren Generation verlief in der Regel recht heterogen, oft in mehreren abrupt endenden Phasen, die zum Teil wenig miteinander zu tun hatten. Querverschiebungen in entfernte Bereiche waren an der Tagesordnung. Firmeninterne Stellenmärkte sind ein Produkt jener Zeit. Eine Normkarriere wie bei der älteren Generation gab es jetzt nicht mehr. Es gab Chemiker, die als Basischemiker ohne jede Charge in Pension gingen. Der Median schaffte es zwar noch bis etwas über den Prokuristen hinaus. Es gab selbstverständlich auch in der mittleren Generation noch weiterführende Karrieren. Indessen, ein Vizedirektor von 1980 war bei weitem nicht mehr dasselbe wie ein Vizedirektor von 1950. Zu sehr hatte die Titelinflation gewütet. Es kam jetzt vor, dass einem Vizedirektor drei Mitarbeiter unterstellt waren und dass ein stellvertretender Direktor Sekretär eines Direktors war. Entsprechend flacher verliefen auch die Salärentwicklungen.

Obwohl sich der Chemiker der mittleren Generation am Ende seiner Laufbahn salärmässig kaum von entsprechenden Inhabern von Vertrauensstellungen in anderen Branchen unterschied, da diese den Rückstand zur chemischen Industrie inzwischen aufgeholt hatten, lebte er dennoch in durchaus bequemen wirtschaftlichen Verhältnissen. Nicht zuletzt wardies darauf zurückzuführen, dass er in der Regel noch in den 50er oder 60er Jahren ein ansprechendes Einfamilienhaus an bevorzugter Wohnlage erwerben konnte, dessen Finanzierung zu äusserst vorteilhaften Bedingungen durch den Arbeitgeber erfolgte. Gegen Ende seines Berufslebens war dann die hypothekarische Verschuldung entweder abgetragen oder infolge Inflation auf ein kaum noch belastendes Niveau abgesunken. Zudem war der Chemiker der mittleren Generation seiner sozialen Herkunft entsprechend meistens durch Erbschaft in den Besitz von Vermögen gelangt, so dass er seinen Ruhestand ohne Einschränkungen geniessen konnte.

Insgesamt hatte es der Chemiker der mittleren Generation jedoch nicht einfach. Seine hochgesteckten beruflichen Erwartungen erfüllten sich oft nicht oder dann mussten Berufsziele zurückgesteckt werden. Ernüchterung, gelegentlich auch Enttäuschung gehörte zu seinem Berufsbild. Er musste als direkt Betroffener die Demontage des Chemikers vom umworbenen, verklärten Helden zum Unkostenfaktor miterleben. Ein entsprechend hoher Anteil hätte denn auch, wenn er nochmals hätte von vorn anfangen können, nicht mehr den Beruf des Chemikers ergriffen.
Fast keiner motivierte seine Kinder, Chemie zu studieren.

\section{Das Berufsbild der jüngeren Chemikergeneration}

\subsection{Herkunft und Berufswahl}

Die jüngere Chemikergeneration, die nach der Ölkrise ab 1973 in das Berufsleben trat, hatte es demgegenüber wieder wesentlich einfacher, sich im Beruf zurechtzufinden, obwohl gerade der Anfang nicht ganz unproblematisch war. Der Zugang zum Beruf war jetzt zwar weit geöffnet. Chemiker entstammten nun vorwiegend dem unteren Mittelstand oder wirtschaftlich schwächeren Schichten. Söhne (oder Töchter) von Arbeitern, Magazinern, Polizisten, Malern, Tapezierern, Bäckem, Schneidern, Köchen, Laboranten und Bauern entdeckten nun den Beruf. Damit vergrösserte sich das Potential zur Rekrutierung von Chemikern theoretisch um ein Vielfaches. Der weitgehende Wegfall rein wirtschaftlicher Motivation zur Berufswahl, die Eröffnung attraktiver alternativer Berufsmöglichkeiten in Berufsfeldern, die ähnliche Fähigkeiten und Neigungen voraussetzten, so z.B. der Informatik, die in den 70er und 80er Jahren Massen von Studierenden anzog, die früher vielleicht Chemiker geworden wären, sowie nicht zuletzt das angeschlagene ökologische Image des Chemikerberufes führten jedoch dazu, dass das theoretische Rekrutierungspotential längst nicht ausgeschöpft werden konnte, sondern dass die Zahl der Chemiestudierenden sogar stark rückläufig wurde.

Diese Ausgangslage wirkte sich in dreierlei Hinsicht positiv auf das Berufsbild des Chemikers aus. Zum einen wurde der Chemikerberuf jetzt zum typischen Aufsteigerberuf. Für den Sohn des Laboranten und den Enkel des Chemiearbeiters war der Aufstieg zum Chemiker auch unter stark veränderten Rahmenbedingungen ein Erfolgserlebnis und seine Berufserwartungen waren nicht euphorisch, sondern realistisch. Zum zweiten führten die sinkenden Ausbildungszahlen zu einem tendenziell verbesserten Stellenmarkt für Chemiker. Zumindest reicht die heutige Produktion von Hochschulchemikern und Chemikern HTL bei weitem nicht aus, den mindestens doppelt so grossen Bedarf zu decken.Zum dritten stieg der Anteil derjenigen, die aus echter Affinität zum Beruf, d.h. aus einer inneren Berufung heraus zur Chemie stiessen, markant an. Die vielen Mitläufer und Glücksritter der 50er und $60 \mathrm{erJahre} \mathrm{sind} \mathrm{heute} \mathrm{verschwunden.} \mathrm{Wenn}$ nur noch jeder 50 . Studienanfänger Chemie wählt, so sollte man nicht darüber lamentieren, sondern sich darüber freuen, dass es mit grösserer Wahrscheinlichkeit als früher die richtigen sind.

\subsection{Studium}

Die Chemikerausbildung wies in den letzten 30 Jahren einen deutlichen Trend zur Verschulung auf. Damit wurde zwar sichergestellt, dass das Studium ein echter Full time job war. Anderseits führt jede Verschulung automatisch zu einem nicht übermässigen Schwierigkeitsgrad, da auf das Mittelmass Rücksicht zu nehmen ist. Die Zahl der Studienabbruiche scheint jedenfalls deutlich zurückgegangen zu sein. Im Vergleich zu früher wurde jetzt auch die Ausbildung auf der Promotionsstufe wieder effizienter, da sich einerseits deren Dauer im allgemeinen auf das vertretbare Mass von drei Jahren reduzierte, anderseits die Kandidaten wieder vermehrt zu selbständigem wissenschaftlichem Arbeiten angeleitet wurden.

Insgesamt haben sich die Ausbildungszeiten stabilisiert oder gar etwas zurückgebildet. Die den Durchschnitt verzerrenden Ausreisser mit zehn bis zwölf und mehr Studienjahren kommen kaum noch vor. Anderseits ist das Postdoktorat, besser noch zwei, für angehende Forschungschemiker ein Muss geworden, so dass sich am Durchschnittsalter ins Berufsleben eintretender Industriechemiker kaum etwas geändert hat. Diese Situation ist nicht nur aus der Sicht der angehenden Chemiker, sondern auch aus Sicht der Industrie wenig befriedigend.

\subsection{Eintritt in \\ das Berufsleben}

Der Stellenmarkt war für den Chemiker der jüngeren Generation der Situation der Branche entsprechend generell zäh, besonders natürlich in der 2 . Hälfte der 70er Jahre. Er versiegte jedoch nie völlig. Die Anfangssaläre lagen nun insofern etwas tiefer als zuvor, als sie näher beim Anderthalbfachen eines Chemiearbeiterlohnes (jetzt GAV-Lohn genannt) lagen, als beim Doppelten. Neu war im Vergleich zu früher, dass Chemiker nun nicht mehr primär für institutionalisierte Funktionen, sondern gezielt für Projekte mittelfristiger Dauer gesucht wurden. In den Vordergrund traten nun oft die Spezialkenntnisse des Kandidaten, d.h. es erwies sich als ausschlaggebend, ob dieser schon bei der Wahl des Doktorvaters und des Dissertationsthemas den richtigen 'Riecher' gehabt hatte. Wer sich schon vor 1980 auf das zunächst vielleicht wenig spektakuläre Problem der Reinigung von Enzymen spezialisiert hatte, war bald ein gesuchter Spezialist, während derjenige, der sich während der Dissertation mit zumindest für Theoretiker spektakulären Problemen beschäftigt hatte, d.h. mit 'Art 
pour l'art', jetzt keine angemessene Stelle fand.

Der Bedarf an Forschungschemikem war überhaupt rückläufig. Zum einen reduzierte sich der Anteil der Chemiker an der pharmazeutischen Forschung von ursprünglich über $90 \%$ auf einen Drittel. Zum anderen tötete die Reorganisationswelle der 70er und 80er Jahre manches Entwicklungsgebiet endgültig ab zu Gunsten von Rationalisierungsmassnahmen. Die Reduktion der Produktionskosten eines Wirkstoffes von 60000 auf $6000 \mathrm{Fr} . / \mathrm{kg}$ versprach eine viel sicherere Rendite als eine Investition in eine unsichere Innovation. Demzufolge eröffneten sich für Verfahrensforscher oft bessere Berufsmöglichkeiten als für Forschungschemiker. Relativ leicht fanden auch Analytiker eine Stelle, da Sicherheitsauflagen, Umweltschutz und Qualitätskontrollen völlig neue Dimensionen annahmen. Die Forschung in zukunftsträchtigen Gebieten wurde demgegenüber gelegentlich mit unterkritischer Masse betrieben, so dass später teure Akquisitionen erforderlich wurden mit der Nebenwirkung, dass sich ganze Forschungsgebiete schwerpunktmässig aus der Schweiz ins Ausland verlagerten.

\subsection{Berufsalltag}

Der Berufsalltag des Chemikers der jüngeren Generation sieht in vielerlei Hinsicht anders aus als früher. War der Chemiker der älteren Generation unter wenig Dutzend Berufskollegen, arbeitete der Chemiker der mittleren Generation als einer unter Hunderten, der Chemiker der jüngeren Generation als einer unter Tausend oder mehr, d.h. als anonymer Mitarbeiter einer Grossunternehmung. Seine Tätigkeit spielt sich jetzt vorrangig sitzend ab. Er braucht also einen bequemen Bürostuhl. Der Chemiker der älteren Generation hatte noch stehend gearbeitet und es stand ihm auch nur ein unbequemer hochbeiniger Laborhocker zur Verfügung. Die heute noch übliche kleine Zelle als Annex des Labors scheint eine steingewordene Reminiszenz einer überholten Berufswirklichkeit zu sein. Im Vergleich zu den meisten anderen Berufen verfügt der Chemiker über einen unangemessenen Arbeitsplatz, der kaum Behaglichkeit ausstrahlt und Wohlbefinden erzeugt, eine von Planungsstellen noch nicht realisierte Folge des Berufsbildwandels.

Wenn heute der Autor als Vertreter der mittleren Chemikergeneration ein Forschungslabor betritt, so fällt ihm in erster Linie die beinahe klösterliche Atmosphäre auf: kein laufendes Rührwerk, kein Blubbern, kein kochendes Ölbad, keine laufende Destillation, kein Lösungsmittelduft, sondern alles wirkt fast beängstigend auf- geräumt. Bei den vielen Instrumenten sind die Hauptschalter alle auf 'off'. Viele schlummern unter ihren Plastikhüllen. Das Labor erscheint nicht als Arbeitsstätte, sondern als Bereitschaftsstätte. Es ist alles da für den Fall, dass es etwas zu tun gäbe. DerChemiker liest unterdessen Zeitschriften, studiert interne Berichte, verfasst selber solche oder befindet sich an einer Sitzung. Die Arbeit des Chemikers ist unsichtbar geworden, scheint sich im wesentlichen in seinem Kopf abzuspielen.

Fast alle Chemiker, mit denen der Autor in jüngster Zeit Kontakt hatte, sind mit Herz und Seele in ihrem Beruf tätig, ähnlich wie das heute etwa auch bei Informatikern beobachtet werden kann. Beides sind Berufe, bei denen es in erster Linie auf das Problemlösungspotential des Individuums ankommt. Die abstrakte Problemlösung bringt die berufliche Befriedigung, nicht mehr das marktgängige Handelsprodukt. Und da es unendlich viele Probleme zu lösen gibt, ist auch das Potential für Erfolgserlebnisse unerschöpflich.

Nun leben jedoch Unternehmungen nicht von Problemlösungen allein, sondern von Cash Flow und Ertrag. Beides sind Phänomene, die von der Welt des Chemikers je nach Einsatzgebiet mehr oder weniger weit entfernt sind. Daraus ergibt sich denn auch ein gewisses Unbehagen des heutigen Industriechemikers. Seine Arbeit ist letztlich fremdbestimmt. Ob sein Arbeitsgebiet gefördert, beschnitten oder gar gestrichen wird, entscheiden irgendwelche 'Heinis' in einer fernen Marketingabteilung, die ja aus Sicht des Chemikers von Tuten und Blasen keine Ahnung haben. Im Vergleich zur Arbeitswelt des Chemikers der älteren Generation hat sich daher die Beziehung zwischen Labor und Verkauf diametral verändert. In der Marketingabteilung hängen heute lauter Damoklesschwerter. Angesichts des extrem hohen Spezialisierungsgrades eines Forschungschemikers kann ein Marketingentscheid eine abrupte Zäsur in seiner beruflichen Laufbahn bewirken, denn es ist nahezu ausgeschlossen, dass ein Forschungschemiker in ein weit entferntes neues Forschungsgebiet eindringen kann.

Überhaupt kommt sich der heutige Chemiker gelegentlich als fünftes Rad am Wagen vor. Dies erläuterte dem Autor vor kurzem ein Chemiker bildhaft wie folgt: 'In unserer Firma geben Heere von Chemikern ihr bestes und produzieren zusammen einen Gewinn von 500 Mio. Fr. In der Finanzabteilung sitzt eine kleine Schar von Spezialisten, die eine weitere Milliarde Gewinn produziert. Wozu braucht es uns da eigentlich noch?' Übersehen hat dieser Chemiker dabei allerdings die Tatsache, dass zumindest die Ausgangssub- stanz, mit welcher die Finanzspezialisten arbeiten, zuvor von Chemikern miterschaffen werden musste.

Die Einbettung in ein Grossunternehmen wird vom Chemiker ambivalent empfunden. Zum einen realisiert er, dass er als Spezialist nur in der Grossunternehmung sicher aufgehoben ist. Anderseits liebäugelt er sehr wohl mit den verlockenden Möglichkeiten eines Ausbruchs in unternehmerische Selbstverantwortung und träumt von seiner eigenen kleinen Firma oder von einer Consulting-Tätigkeit. Mit Faszination registriert er das Aufblühen Tausender von Klein- und Mittelbetrieben im Bereich der Biotechnologie vorwiegend in den USA. Insgeheim aber weiss er, dass es nur ein Traum ist, dass er wahrscheinlich eben doch bleiben wird und seine Ersparnisse lieber in ein sicheres Einfamilienhaus investiert als in ein riskantes Unternehmen.

\subsection{Karriere und gesellschaftliche Stellung}

Der heutige Chemiker weiss und rechnet damit, dass sein Berufsleben nicht geradlinig verlaufen wird, dass er nicht 40 Jahre lang im selben Gärtlein wirken wird und dass er jederzeit mit Reorganisationen und Versetzungen konfrontiert werden kann. Viele möchten allerdings auch nicht jahrzehntelang dasselbe tun müssen, sondern wünschen sich aktiv durchschnittlich etwa alle zehn Jahre eine neue Herausforderung. Die Erwartungen hinsichtlich Karriere scheinen eher zurückhaltend zu sein, was angesichts der flachen und straffen Organisationen der $90 \mathrm{er}$ Jahre auch realistisch ist. DerGruppen-oder Bereichsleiter ist vielfach die obere Grenze einer Karriereerwartung. 'Auf dem Höhepunkt meiner Karriere möchte ich $125000 \mathrm{Fr}$ verdienen' sagte kürzlich ein Chemiker mit einigen Jahren Praxis. Real entspricht dies vom Zeitpunkt des Eintritts an gerechnet einer Steigerung von etwa $50 \%$, nicht zu vergleichen mit der theoretisch möglichen Versechzigfachung des Einkommens eines Chemikers der älteren Generation. Die realistische Karriereerwartung hat allerdings den Vorteil, dass das Ziel mit an Sicherheit grenzender Wahrscheinlichkeit erreicht werden wird. Möglicherweise wird es sogar übertroffen. 